\title{
Conocimiento y actitud del odontólogo frente al manejo del tabaquismo: estudio comparativo entre España, Italia y Venezuela
}

\section{The participation of dentists in smoking cessation: comparative study of Venezuelan, Spanish and Italian dentists}

\author{
Villarroel Dorrego M*, Bascones-Martínez A**, Pérez González E*, Lauritano D***
}

\section{RESUMEN}

Introducción: El Odontólogo es capaz de ejercer un gran impacto en el tabaquismo a través de su contacto con los pacientes y la detección precoz de lesiones bucales. El objetivo de este estudio fue determinar el conocimiento y la actitud de los odontólogos frente al tabaquismo en tres países distintos.

Materiales y métodos: Se realizó una encuesta a 293 odontólogos, 93 de ellos localizados en Caracas (OV), 90 con práctica odontológica en Madrid (OE) y 110 en Milán (OI).

Resultados: 46 OE y $22 \mathrm{OI}$ indicaron ser fumadores, mientras que tan solo $10 \mathrm{OV}$ admitieron ser fumadores. $80(86,02 \%)$ OV, $66(73,33 \%)$ OE y todos los OI refirieron registrar en la historia datos relacionados con tabaquismo en la primera consulta del paciente. La mayoría motiva a sus pacientes a dejar de fumar, sin embargo, 72,04\% (67) OV, 55,55\% (50) OE y 100\% OI no recomiendan ninguna terapia de apoyo. Sólo 7 odontólogos (2,38\%) (2 OV y 5 OE) reconocieron el medicamento Bupropion pero ningún odontólogo lo ha indicado. Finalmente, 137 odontólogos han diagnosticado cáncer bucal, de los cuales 108 eran OI.

Conclusiones: Los odontólogos, independientemente del país, tienen actitudes positivas para participar en el control del tabaquismo, pero no manejan la información relacionada ni ejercen un rol activo en la cesación. Probablemente esta situación se deba a la falta de entrenamiento durante sus estudios de pre y/o postgrado lo cual amerita una revisión de los planes curriculares de Odontología a nivel mundial.

Palabras clave: Control tabaquismo, lesiones bucales por tabaco.

\section{SUMMARY}

Introduction: Health care professionals have an important role in tobacco control. Dentists are capable of having a great impact in smoking cessation due to their regular contact with patients and the rapid detection of oral signs produced by smoking. Unfortunately, there are few dentists actively involved in smoking control. The aim of this study was to investigate various aspects of dentists' beliefs and practices with respect to smoking cessation. Materials $E$ Methods: A questionnaire about smoking cessation was filled in person by 293 dentists. 93 dentists were from Caracas-Venezuela (VD), 90 were from Madrid-Spain (SD) and 110 from Milan-Italy (ID). Results: 46 SD and 22 ID were smokers. Only 10 VD admitted to smoke. 80 (86.02\%) VD, 66 (73.33\%) SD and $100 \%$ of ID said that they recorded smoking status during the first consultation. However, none routinely update their records on smoking status. The majority of respondents encourage their patients to stop smoking, though, 72.04\% (67) VD, 55.55\% (50) SD and 100\% ID do not recommend or prescribe any cessation therapy. Just 7 (2.38\%) of all dentists had heard about the use of Bupropion, however, they had never indicated. Finally, 137 dentists (108 ID) have diagnosed oral cancer in their patients.

\footnotetext{
* $\quad$ Universidad Central de Venezuela.

** Universidad Complutense de Madrid.

*** Universidad de Milán.
} 
Conclusions: Dentists have positive attitudes to being involved in smoking cessation interventions, but they do not manage all the information related to it. It is probably due to a lack of training or education during their undergraduate and postgraduate studies. Further studies should evaluate inclusion of smoking cessation instruction in Venezuelan, Spanish and Italian dentistry curriculum.

Key words: Tobacco control, oral lesions tobacco.

Fecha de recepción: Marzo 2008.

Aceptado para publicación: Abril 2008.

Villarroel Dorrego M, Bascones-Martínez A, Pérez González E, Lauritano D. Conocimiento y actitud del odontólogo frente al manejo del tabaquismo: estudio comparativo entre España, Italia y Venezuela. Av. Odontoestomatol 2009; 25 (4): 209-213.

\section{INTRODUCCIÓN}

El uso de tabaco en sus distintas formas ha sido claramente asociado a alteraciones sistémicas como cáncer y enfermedades cardiovasculares. En la cavidad bucal, es causa irrefutable de leucoplasia y eritroplasia (1) y ha sido relacionado con halitosis, enfermedad periodontal, pigmentaciones de la mucosa $\mathrm{y} / \mathrm{o}$ restauraciones y hasta fracaso de tratamientos con implantes dentales (2).

Entre los profesionales de la salud, es el equipo odontólogo/higienista el que tiene mayor oportunidad de intervenir en la cesación tabáquica de la población, ya que la práctica odontológica comprende un contacto regular con los pacientes y los signos bucales producidos por el tabaco son evidentes para estos profesionales (3). Además, los odontólogos pueden causar un gran impacto en prevención de tabaquismo dada su constante relación con la comunidad.

Lamentablemente la intervención de los odontólogos en el proceso de prevención y cesación del tabaquismo es muy poca y esto se deba posiblemente a la falta de capacitación de los estudiantes en los programas de odontología a nivel mundial (4-6).

El objetivo del presente trabajo fue determinar las competencias que poseían los odontólogos en relación manejo del tabaquismo en tres países distintos.

\section{MATERIALES Y MÉTODOS}

Se realizó una encuesta a 293 odontólogos, en Caracas (Venezuela) (OV), en Madrid (España) (OE) y en Milán (Italia) (OI). Fueron seleccionados profesionales con vinculación con la academia, con práctica privada, o con práctica privada exclusivamente. Fueron recolectados datos en relación a las siguientes variables:

a. Género, edad y nivel de postgrado.

b. Nivel de conocimientos en relación al diagnóstico y tratamiento del tabaquismo.

c. Práctica de dichos conocimientos en la clínica.

d. Participación en el diagnóstico o detección de cáncer bucal.

Los datos fueron organizados para su análisis estadístico descriptivo usando SPSS versión 11.0.

\section{RESULTADOS}

Doscientos noventa y tres odontólogos (93 OV, 90 OE y 110 OI) completaron la encuesta de 300 instrumentos aplicados (97,6\% respuesta). 170 odontólogas $(58,02 \%)$ y 123 odontólogos $(41,98 \%)$ en total distribuidos entre los países de la siguiente forma: $63(67,74 \%)$ mujeres y $30(32,26 \%)$ hombres OV; $60(66,67 \%)$ mujeres y $30(33,33 \%)$ hombres OE y, finalmente, $47(42,73 \%)$ mujeres y $63(57,27 \%)$ hombres $\mathrm{OI}$, con edades comprendidas entre 23 y 
72 años (OV media edad 30,88 $\pm 8,52$ años; OE media edad 27,96 $\pm 7,005$ años, OE media edad 40,4 $\pm 12,32$ años).

Ciento sesenta y tres Odontólogos (55,63\% población estudiada) refirieron poseer estudios de postgrado, distribuidos entre los países de la siguiente manera: 54 OV en las áreas de Operatoria y Estética, Medicina Bucal, Implantes, Endodoncia, Cirugía Bucal, Periodoncia, Estomatología integral del adulto, Docencia universitaria, Odontopediatría, Ortopedia funcional, Patología bucal, Prótesis. 64 OE en las áreas de Implantes y Estética, Periodoncia, Prótesis, Medicina Bucal, Odontología Conservadora, Endodoncia, Cirugía Bucal, Ortodoncia, Sanitaria, Cirugía Maxilofacial, Odontopediatría y Forense. $45 \mathrm{OI}$ en las áreas de Odontoestomatología y Ortodoncia.

Cuarenta y seis OE (51,11\%) y 22 (20\%) OI indicaron ser fumadores, mientras que tan solo 10 (10.75\%) OV admitieron fumar. Todos los fumadores consumían el tabaco de manera inhalada en cigarrillos.

Ochenta (86,02\%) OV, 66 (73,33\%) OE y todos los OI refirieron preguntar y registrar en la historia datos relacionados con tabaquismo en la primera consulta del paciente. La mayoría motiva a sus pacientes a dejar de fumar, sin embargo, 72,04\% (67) OV, 55,55\% (50) OE y $100 \%$ OI no recomiendan ninguna terapia de apoyo. Entre las recomendaciones indicadas a los pacientes para dejar de fumar los odontólogos prescriben parches y chicles de nicotina, uso de caramelos sin azúcar, uso de hilo dental y cepillado, terapias psicológicas o referencias a otros médicos, entre otros (Tabla 1).

Sólo 7 odontólogos (2,38\%) (2 OV y 5 OE) reconocieron el medicamento Bupropion pero ninguno lo había indicado. Finalmente, 137 odontólogos han diagnosticado cáncer bucal, de los cuales 108 eran OI.

\section{DISCUSIÓN}

Los odontólogos, independientemente de su país de origen, tienen una actitud positiva para participar activamente en el control del tabaquismo. Estudios previos coinciden en que el odontólogo intenta mo- tivar a su paciente para cesar el consumo del tabaco en cualquiera de sus formas pero no tiene buen conocimiento sobre terapias cognitivas, conductuales o farmacológicas utilizadas para el tratamiento del tabaquismo (7-10).

Los odontólogos están conscientes de su responsabilidad en el tratamiento del tabaquismo y su prevención, sin embargo no se sienten lo suficientemente preparados para brindar dicha terapéutica. Esta situación es debido a la falta de entrenamiento a nivel de pregrado y/o postgrado y al poco énfasis que se hace al tabaquismo y el manejo del paciente en forma integral durante los estudios de odontología.

La incorporación del tabaquismo en la plenitud de su estudio debe ser reforzada en el currículo de las escuelas de Odontología, e inclusive debe ser tema incorporado en los estudios de higienista dental (5). Edwards et al. mostraron que higienistas dentales tienden a recomendar mucho más a los pacientes la cesación del tabaquismo, e inclusive los pacientes sienten más confianza y comparten mayor tiempo de consulta con higienistas que los odontólogos, sin embargo su adiestramiento en el área les impide dar un efectivo tratamiento al paciente (10).

Para un verdadero impacto en el problema de tabaquismo se ha sugerido enfáticamente la inclusión de los siguientes aspectos en el currículo de odontología: Enseñanza de los efectos biológicos producidos por el uso de tabaco, historia del tabaco y aspectos socio-culturales asociados a su uso, prevención y tratamiento del tabaquismo y desarrollo de habilidades clínicas para el manejo de pacientes con tabaquismo (6).

Otros aspectos que han sido considerados en la falta de participación del odontólogo en la cesación del uso del tabaco son la falta de tiempo durante las consultas y la poca remuneración en términos del procedimiento (11). La consulta para dejar de fumar no debe ser un aspecto extra de la consulta odontológica, debe planificarse para tal fin y darle el tiempo que se estipule necesario para de esta forma garantizar un porcentaje mayor de éxito (12).

La inclusión del odontólogo en el equipo de salud comunitaria, en cualquiera de sus niveles, es hoy 


\section{TABLA 1.- RESUMEN DE RESPUIESTAS DE LOS ODONTÓLOGOS}

\begin{tabular}{|c|c|c|c|}
\hline Preguntas & Respuestas OV & Respuestas OE & Respuestas OI \\
\hline $\begin{array}{l}\text { ¿Qué preguntas realiza } \\
\text { usted relacionadas con } \\
\text { el tabaquismo a su } \\
\text { paciente? }\end{array}$ & $\begin{array}{l}\text { No pregunta } 13,98 \% \\
\text { Si es fumador } \\
\text { Cantidad y frecuencia } \\
\text { Tipo de tabaco } \\
\text { Inicio del consumo } \\
\text { Razones del consumo } \\
\text { Efectos consumo }\end{array}$ & $\begin{array}{l}\text { No pregunta } 26,67 \% \\
\text { Si es fumador } \\
\text { Cantidad y frecuencia } \\
\text { Tipo de tabaco } \\
\text { Inicio del consumo } \\
\text { Razones del consumo } \\
\text { Antecedentes tabáquicos } \\
\text { familiares }\end{array}$ & $\begin{array}{l}\text { No pregunta } 0 \% \\
\text { Si es fumador } \\
\text { Cantidad } \\
\text { Frecuencia } \\
\text { Tipo de tabaco }\end{array}$ \\
\hline $\begin{array}{l}\text { ¿Qué datos relacionados } \\
\text { con el tabaquismo } \\
\text { recolecta usted en la } \\
\text { historia clínica? }\end{array}$ & $\begin{array}{l}\text { No indica nada en } \\
\text { la historia } 13,98 \% \\
\text { Si es fumador } \\
\text { Cantidad y frecuencia } \\
\text { Tipo de tabaco } \\
\text { Lesiones mucosas } \\
\text { asociadas }\end{array}$ & $\begin{array}{l}\text { No indica nada en } \\
\text { la historia } 26,67 \% \\
\text { Si es fumador } \\
\text { Cantidad y frecuencia } \\
\text { Tipo de tabaco } \\
\text { Lesiones mucosas } \\
\text { asociadas }\end{array}$ & $\begin{array}{l}\text { No indica nada } \\
\text { en la historia } 0 \% \\
\text { Si es fumador } \\
\text { Cantidad y frecuencia } \\
\text { Tipo de tabaco } \\
\text { Lesiones mucosas } \\
\text { asociadas }\end{array}$ \\
\hline $\begin{array}{l}\text { ¿Motiva usted a su } \\
\text { paciente para dejar } \\
\text { de fumar? }\end{array}$ & $\begin{array}{l}\text { Sí } 98 \% \\
\text { No } 2 \%\end{array}$ & $\begin{array}{l}\text { Si } 99 \% \\
\text { No } 1 \%\end{array}$ & $\begin{array}{l}\text { Si } 100 \% \\
\text { No } 0 \%\end{array}$ \\
\hline $\begin{array}{l}\text { ¿Qué medios indica } \\
\text { para la cesación del } \\
\text { tabaquismo? }\end{array}$ & $\begin{array}{l}\text { Ninguno } 72,04 \% \\
\text { Reemplazo nicotina } \\
\text { (Parches y chicles) } \\
\text { Refiere médico, } \\
\text { psicólogoo especialista } \\
\text { tabaquismo } \\
\text { Terapia conductual } \\
\text { Control y eliminación } \\
\text { de ansiedad } \\
\text { Terapia con Bupropion } \\
\text { Cepillado, uso de } \\
\text { enjuague e hilo dental } \\
\text { Chicles o caramelos } \\
\text { sin azúcar } \\
\text { Ejercicios miofaciales } \\
\text { Hipnosis, homeopatía }\end{array}$ & $\begin{array}{l}\text { Ninguno 55,55\% } \\
\text { Reemplazo nicotina } \\
\text { (Parches y chicles) } \\
\text { Refiere médico, } \\
\text { psicólogo o especialista } \\
\text { tabaquismo. Consulta } \\
\text { farmacéutico } \\
\text { Terapia conductual } \\
\text { Terapia con Bupropion } \\
\text { Fuerza de voluntad } \\
\text { Cepillado, uso de } \\
\text { enjuague e hilo dental } \\
\text { Caramelos de menta } \\
\text { Lectura de libro } \\
\text { Deporte }\end{array}$ & $\begin{array}{l}\text { Ninguno } 100 \% \\
\text { Fuerza de voluntad }\end{array}$ \\
\hline
\end{tabular}

día una obligación más que una opción, por lo que los cambios curriculares de las escuelas de odontología deben ser planificados para dar respuesta a este perfil que demanda la sociedad. La adquisición de competencias relacionadas al manejo del tabaco es, sin duda, congruente con la necesidad social y los cambios actuales del perfil del profesional de la salud. 


\section{BIBLIOGRAFÍA}

1. Napier SS, Speight PM. Natural history of potentially malignant oral lesions and conditions: an overview of the literature. J Oral Pathol Med. 2008;37(1):1-10.

2. Tobacco and oral diseases-report of EU Working Group, 1999.EU-Working Group on Tobacco and Oral Health. J Ir Dent Assoc 2000;46(1): 12-9.

3. Jonson NW. The role of the dental team in tobacco cessation. Eur Dent J Edu 2004; 8(Suppl 4):18-24.

4. Warnakulasuriya KA, Johnson NW. Dentists and oral cancer prevention in the UK: opinions, attitudes and practices to screening for mucosal lesions and to counselling patients on tobacco and alcohol use: baseline data from 1991 Oral Dis 1999;5(1):10-4.

5. Fried JL, Rubinstein-DeVore L. Tobacco use cessation curricula in the U.S. dental schools and dental hygiene programs. J Dent Educ 1990 Dec; 54(12):730-5.

6. Ramseier CA, Christen A, McGowan J, McCartan B, Minenna L, Ohrn K, Walter C. Tobacco use prevention and cessation in dental and dental hygiene undergraduate education. Oral Health Prev Dent. 2006;4(1):49-60.
7. John JH, Thomas D, Richards D. Smoking cessation interventions in the Oxford region: changes in dentists' attitudes and reported practices 1996-2001. Br Dent J 2003;195(5): 270-5.

8. Albert DA, Severson H, Gordon J, Ward A, Andrews J, Sadowsky D. Tobacco attitudes, practices, and behaviors: a survey of dentists participating in managed care. Nicotine Tob Res. 2005;7 Suppl 1:S9-18.

9. Wyne AH, Chohan AN, Al-Moneef MM, Al-Saad AS. Attitudes of general dentists about smoking cessation and prevention in child and adolescent patients in Riyadh, Saudi Arabia. J Contemp Dent Pract 2006; 7(1):35-43.

10. Edwards D, Freeman T, Roche AM. Dentists' and dental hygienists' role in smoking cessation: an examination and comparison of current practice and barriers to service provision. Health Promot J Austr. 2006;17(2):145-51.

11. Stacey F, Heasman PA, Heasman L, Hepburn S, McCracken GI, Preshaw PM. Smoking cessation as a dental intervention-views of the profession. Br Dent J. 2006; 201(2):109-13.

12. Tomar SL. Dentistry's role in tobacco control.J Am Dent Assoc. 2001;132 Suppl:30S-35S. 